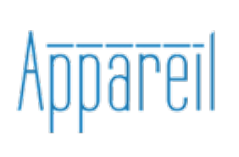

Appareil

$13 \mid 2014$

Trauma et création

\title{
Pour une esthétique benjaminienne : couleur, pore, pli
}

Denis Skopin

\section{(2) OpenEdition}

Journals

Édition électronique

URL : http://journals.openedition.org/appareil/2017

DOI : 10.4000/appareil.2017

ISSN : 2101-0714

Éditeur

MSH Paris Nord

Référence électronique

Denis Skopin, «Pour une esthétique benjaminienne : couleur, pore, pli », Appareil [En ligne], 13 | 2014, mis en ligne le 23 juillet 2014, consulté le 30 juillet 2020. URL : http://journals.openedition.org/ appareil/2017; DOI : https://doi.org/10.4000/appareil.2017

Ce document a été généré automatiquement le 30 juillet 2020.

\section{(c) $($ ) $(9)$}

Appareil est mis à disposition selon les termes de la Licence Creative Commons Attribution - Pas d'Utilisation Commerciale - Pas de Modification 4.0 International. 


\title{
Pour une esthétique benjaminienne : couleur, pore, pli
}

\author{
Denis Skopin
}

\section{Introduction}

1 Le texte de Jean-Louis Déotte sur Germain Rœsz, «Entre trauma et coma », peut être rapproché d'un manifeste artistique, car il renferme tout un programme pour une peinture à venir. Mais il peut également se lire comme un vaste projet de recherche, parce qu'il nous offre une perspective où nous placer pour l'analyse de l'art contemporain.

2 D'abord, le texte de Déotte reprend la grande discussion sur l'origine de l'œuvre d'art qui, au xx $x^{e}$ siècle, a opposé Heidegger d'un côté, Benjamin et Adorno (puis Schapiro et Derrida) de l'autre. Déotte met au centre de sa lecture les notions de coma et d'anamnèse, notions qui ont en commun de relever d'une certaine physiologie. Selon Déotte, ce sont les processus "physiologiques» au sens large du terme qui sont à l'origine de l'œuvre d'art. Il s'agit des accidents de santé qui viennent interrompre la vie quotidienne, qui brisent son unité, qui nous arrachent à notre existence de tous les jours. Sans cela, il n'y aurait pas d'art. Ce n'est pas la mémoire qui est à l'origine de l'art, mais la perte de la mémoire, un choc, un trauma, une crise d'épilepsie.

3 D'ailleurs, la notion de coma est à comprendre au sens large. Cet état n'est pas dû seulement à une maladie; la cause de l'interruption dont il s'agit peut être différente, celle-ci pouvant être provoquée par une solitude ou un isolement. Un état pareil au coma est décrit dans Le Différend de Lyotard et il est sous-entendu par «la fin de l'expérience » chez Benjamin. Le « coma » dont il s'agit est donc un état universel, une éclipse de la conscience qui arrive à chacun à certains moments de sa vie ${ }^{1}$.

Une fois apparu, l'art ne peut pas exister par lui-même. Les traces ne suffisent pas pour qu'il y ait de l'art. Pour exister, l'art a besoin d'un support. Le deuxième grand thème de la lecture de l'œuvre de Rœsz par Déotte, c'est celui des appareils et de l'appareillage. Les appareils sont des intermédiaires techniques complexes (camera obscura, appareil 
photographique, caméra et autres appareils de prise de vues apparus plus récemment), qui participent à la création artistique, qui donnent aux arts leur assiette. Si les appareils sont d'origine technique, leurs effets débordent la technicité étroite et se font sentir dans plusieurs sphères, y compris le domaine de l'art.

La deuxième thèse de Déotte est la suivante : la peinture de Rœsz est rendue possible par le cinéma, elle est appareillée par l'appareil cinématographique: "les flux cinématographiques privilégient certes le mouvement (poursuites, road movies, etc.), mais s'imposent picturalement par des extraits verticaux de bandes passantes accrochées-là attendant un hypothétique montage $»^{2}$.

Une autre thèse avancée par Déotte, c'est que certains aspects de l'art de Rœsz se révèlent proches de l'esthétique proposée par Benjamin dans sa première période. C'est cette piste que je me propose d'explorer en détail ici. Il ne s'agit certes pas de voir dans cette peinture l'expression de quelque théorie philosophique, mais, en partant de la thèse de Déotte, d'attirer l'attention sur quelques proximités qui existent entre la pratique picturale de G. Rœsz et certains concepts de W. Benjamin.

7 Ce côté benjaminien de la peinture de Rœsz a plusieurs volets, que je vais évoquer à tour de rôle. Il s'agit de l'emploi de la couleur, des techniques de la compénétration et du plissage, puis du goût pour le poreux. J'aborderai également la problématique du fétichisme qui semble présente à la fois chez Rœsz et chez Benjamin.

\section{Couleur}

Dans le dialogue intitulé L'arc-en-ciel, où Benjamin traite de l'origine des couleurs, il identifie la couleur au "plus intime de l'imagination" (das innerste der Phantasie). Comme l'indiquent les fragments qui se rattachent au dialogue, il s'agit, pour Benjamin, de construire la théorie de la vision non-intellectuelle, sans concept, ayant lieu avant l'objectivation par l'entendement. Si bien que l'adjectif «pur» qui apparait dans ce fragment et s'applique aux substantifs "réception » et "voir » (das reine Sehen) ${ }^{4}$ est à comprendre dans un sens inverse au sens kantien: la pureté dont il s'agit est une liberté de concepts.

9 Ces réflexions de Benjamin sont à interpréter comme « un signe de la théorie de la vision sans concepts, préobjectivante... Il s'agit pour lui de rechercher des modes de l'intuition "pure" qui précèdent le processus de la production artistique, l'œuvre d'art elle-même : "le pouvoir d'avoir les intuitions (das Anschauen) propre à la fantaisie est un voir (ein Schauen) à l'intérieur du canon, mais qui ne se conforme à celui-ci ; ce qui veut dire [un pouvoir] purement réceptif, non créateur (unschöpferisch)" $»^{5}$. Selon H. Brüggemann, Benjamin reprend l'ancienne querelle entre disegno et colore, dessin et couleur, avec des connotations négatives, qui va de Vasari à Descartes et à Kant ${ }^{6}$. Voici ce qu'écrit Kant :

Les couleurs qui illuminent un dessin appartiennent à l'excitation [zum Reiz]; bien qu'elles puissent rendre plus expressif un objet en lui-même pour la perception [Empfindung], elles ne peuvent pas le rendre digne d'intuition [contemplation, anschauungswürdig] et beau : au contraire, elles sont, dans la plupart des cas, très limitées par ce qui exige la belle forme, et même là où l'excitation est permise, elle n'est qu'anoblie par elles ${ }^{7}$.

Cette tradition dont on connaît l'influence dans le monde des beaux-arts situe la couleur du côté du sensible et la met en dépendance de la forme qui, elle, est perçue par 
la faculté logique et, d'une façon toute naturelle, est rangée dans la sphère des concepts.

à la nature lumineuse de la couleur, Goethe, suite à une série d'expériences optiques effectuées entre 1790 et 1823, arriva à la conclusion que la couleur doit tout autant son existence à une non-lumière, à l'obscurité qui, selon lui, est elle aussi susceptible de colorer un objet. Ainsi le principe de l'objectivité de la couleur se voit-il complété par son contraire : de même qu'elle surgit à la suite de la diffraction de la lumière, la couleur apparaîtrait à la suite d'une extériorisation, d'une projection de notre imagination vers notre extérieur. La couleur serait donc un milieu où se rencontrent et se correspondent l'intérieur et l'extérieur, l'inné et l'acquis, le subjectif et l'objectif ${ }^{11}$. Comme Goethe, Benjamin estime que l'œil humain peut être producteur de couleurs. Avant d'être la caractéristique de l'objet, la présence des couleurs signale que le sujet de la perception a de l'imagination, la proximité de ses «nuages » qui voilent les formes des objets.

14 La peinture de Rœsz peut être qualifiée de «benjaminienne » au sens où elle fait de la couleur son moyen d'expression privilégié.

Sans grand risque de se tromper, on peut dire que cette esthétique a pour son contraire l'art d'Adami comme il est analysé par Derrida. En effet, la ligne constitue le paradigme de la trace. Quand il parle de la cécité de l'art et de la cécité dans l'art, Derrida se réfère toujours à la pratique du dessin, en abandonnant l'analyse de la peinture et de la couleur. Cela s'explique par la place occupée par le dessin, qui ferait partie du langage et de la rhétorique. Chez Adami, artiste "derridien», le trait parle, même s'il ne devient jamais «bavard». Ce trait itinérant, bifurquant, toujours hétérogène anticipe la couleur, qui «n'arrive jamais avant l'arrêt complet du trait moteur $»^{12}$ et dont la fonction reste secondaire.

16 L'œuvre de Rœsz échappe à la sémiologie de l'art. Les couleurs ne forment pas un langage, même si elles peuvent, quand on les prend comme système, avoir une valeur symbolique. C'est pourquoi les couleurs sont impossibles à déconstruire : «comment 
déconstruire des couleurs sans contours, sans frontières, passant de l'une à l'autre, au contraire d'un Adami ?» ${ }^{13}$

Mais le goût pour la couleur ne signifie pas chez Rœsz le rejet de la forme. Il ne s'agit pas ici d'insister sur un terme de l'opposition aux dépens de l'autre. Rœsz veut se sauver « du formalisme, du diktat de la cohérence, de la continuité, de la réponse que devrait l'art à la seule partition contemporaine ». Mais cela ne signifie pas qu'il refuse «la forme et les concepts qu'ont élaborés un certain nombre d'artistes ${ }^{14}$. Ce n'est pas un art brut, c'est un art hybride ou un art déformant, un art du milieu qui opère dans la « zone obscure » (Simondon) entre la forme et la matière.

Blanche aux couleurs oranges

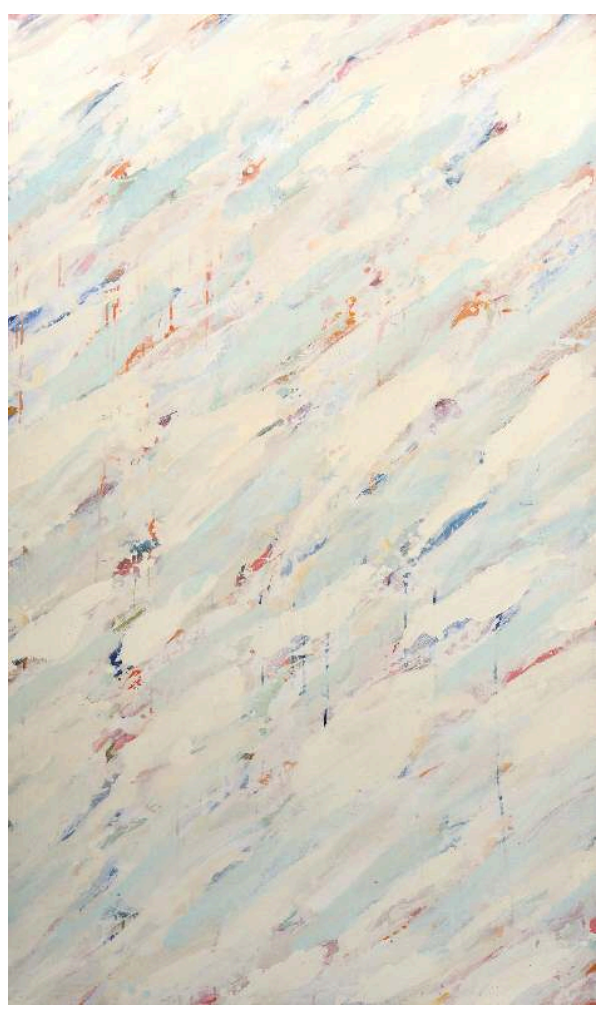

1979

Photo P. Bouvier

\section{Porosité}

L'autre notion benjaminienne qui va nous intéresser, c'est la notion de pore. Benjamin aime les objets poreux et ce modèle est largement présent dans ses écrits consacrés à la fantasmagorie, à côté du modèle textile. L'imagination comme la comprend Benjamin serait une structure poreuse, qui tiendrait d'une pierre volcanique ou d'une éponge.

Avant de passer à l'analyse de la porosité chez Benjamin, il n'est pas inutile de recourir brièvement à la philosophie deleuzienne qui, comme on sait, élabore une taxinomie d'espaces troués, striés, poreux ${ }^{15}$. Il ne s'agira pourtant pas de rechercher des proximités entre deux façons de penser le pore, mais de démontrer leur différence. 

Deleuze en termes d'opposition et d'exclusion. Le corps poreux serait à ses yeux un corps qui substitue un vide à la présence de la matière, comme dans le cas de la chaussette tissée d'une multitude de petits trous. Le pore constitue le contraire du corps, attaque sa structure, inverse les rapports entre le vide et le plein. Il rend la chose absurde, la dépouille de sa valeur « chosale ». L'objet poreux est dans une large mesure un objet dégradé, absurde. Pour Deleuze, la porosité est une maladie rongeant le système profondeur/surface, celui qui est à l'origine de toute hiérarchie. Lorsque Deleuze parle d'un objet couvert de pores, c'est par excellence un objet à deux dimensions, une peau. À la différence de la peau collective lyotardienne qui annule les hiérarchies, la surface deleuzienne est une structure hiérarchisante, une matrice de toute hiérarchisation. sert à caractériser une non-opposition de phénomènes, ayant l'avantage d'établir un rapport entre les contraires. Le pore n'intéresse pas Benjamin comme un vide, mais comme une façon d'établir un rapport entre le vide et le plein. Le modèle du pore que propose Benjamin fait appel aux rapports d'interdépendance des contraires et de leur compénétration (Durchdringung). Chez Benjamin, ce n'est pas la peau ou la surface qui est semée de pores mais un corps tridimensionnel, comme la pierre volcanique de Naples, ville poreuse.

Mais la porosité, au-delà du fait de qualifier un objet matériel quelconque, est une notion qui caractérise les lieux et les situations propices à la fantasmagorie. La porosité (Porosität), le goût pour la porosité, peut caractériser toute une époque, par exemple le $\mathrm{xx}^{\mathrm{e}}$ siècle, étant à l'opposé de la stricte séparation des contraires typique de la vie bourgeoise du siècle précédent ${ }^{16}$.

23 Ainsi la porosité se manifeste-t-elle à travers la conception de la rue dans les pays méridionaux. Les villes comme Naples semblent ignorer la distinction entre l'intérieur et l'extérieur des maisons, qui est en même temps la distinction entre espace privé et espace public. Comme dans les passages urbains de Paris, on y observe «la compénétration enivrée de la rue et de l'appartement $»^{17}$. L'architecture de Naples est poreuse, tout comme la roche sur laquelle la ville est construite. Cette architecture - « la pièce la plus significative de la rythmique d'une communauté $~^{18}$ - est à l'origine de la fantasmagorie qui colore bien des aspects de la vie napolitaine.

S'agissant de l'architecture de cette ville, on constate deux mouvements qui finissent par se rencontrer et faire un: l'intériorisation de l'extérieur et l'extériorisation de l'intérieur. D'un côté, l'intérieur se porte vers l'extérieur. La vie privée sort dans la rue, en la transformant en appartement. La rue devient lieu d'habitation, «l'appartement du collectif $»^{19}$. La ville entière perd de son extériorité pour devenir une espèce d'intérieur. Au lieu de se renfermer dans la coquille de l'appartement, la vie des habitants de Naples se déroule dehors, sous les regards des autres. La conception même de la maison (de l'intérieur) y est inverse de celle qu'on trouve en Europe du Nord. Ce n'est plus un endroit où l'on se met à l'abri du collectif, mais un lieu qui, tout au contraire, participe de la vie du collectif. La salle silencieuse d'une église, qui semble être un espace isolé, privé, est en réalité le dedans de la rue, une poche formée par une sorte de plissement de l'espace public, "l'estuaire baroque d'une vie publique intensifiée $»^{20}$. 
De l'autre côté, c'est la vie collective qui entre dans les appartements. Si, à Naples, il n'y a pas de privé, d'intérieur, c'est aussi parce que le collectif s'approprie le privé, en fait sa modalité. «Il y a une répartition, une porosité et une interpénétration de la vie privée. Ce qui distingue Naples des autres grandes villes, c'est ce qu'elle a en commun avec le village hottentot : toute attitude et action privée est submergée par les flots de la vie sociale $»^{21}$.

Plus un quartier est pauvre, plus la compénétration de l'individuel et du collectif s'y fait sentir. Naples est un immense faubourg, ville sans beaux quartiers, ville qui sert de cadre naturel à l'activité du prolétaire. La pauvreté des Napolitains est inséparable de l'architecture de leur ville. En même temps, c'est cette architecture qui contribue à l'esprit démocratique et carnavalesque de Naples, en fait un lieu propice à la fantasmagorie. Si les beaux quartiers, lieux déserts où prédomine la vie privée, ne sont pas favorables à la flânerie, ce n'est pas le cas des quartiers de Naples, où le flâneur se sent parfaitement à l'aise.

\section{Tissu}

7 Un autre modèle qu'on trouve chez Benjamin et qui pourrait, semble-t-il, servir à caractériser la peinture de Rœsz, c'est le modèle textile.

Dans la Logique du sens, Deleuze décrit la perception d'une chaussette par un schizophrène. Ce dernier appréhende le tissu de la chaussette comme une multitude de petits trous qui ne cessent de s'élargir, de sorte qu'ils menacent la surface de la chaussette de sa dégradation ${ }^{22}$. Selon Deleuze, qui fait usage de la doctrine stoïcienne du lien entre les mots et les états de choses, l'impossibilité de percevoir la surface, qui fait communiquer les mots et les choses, a pour conséquence la disjonction des propositions et des objets auxquels elles doivent se rapporter. Les mots se trouvent détachés des objets et commencent à fonctionner à titre indépendant : étant séparés de leur sens, les mots cessent de signifier et possèdent tout au plus un certain pouvoir de manifester. La surface est une gardienne du sens, un lieu où le sens est généré, si bien que la dégradation de la surface va de pair avec la défaite de la logique. La description de la chaussette sert ici à démontrer la crise de ce système de la production du sens. La folie dont parle Deleuze se manifeste, au niveau de la perception, par une insensibilité aux surfaces, par le fait de ne pas pouvoir les identifier en tant que telles.

Quant au personnage de l'Enfance berlinoise, il se montre, au contraire, extrêmement sensible aux surfaces, à leur géométrie et à la beauté de leur jeu. Si Deleuze est fasciné par les espaces striés et troués, ce n'est pas le cas de Benjamin. Si Benjamin évoque à plusieurs reprises la structure que forme une chaussette retournée sur elle-même, ce n'est pas pour s'interroger sur les rapports entre la surface et la profondeur, responsables de la logique, mais afin de trouver un nouveau rapport entre le dehors et le dedans ${ }^{23}$. Ce qui fascine Benjamin dans cette structure dont, selon lui, on ne peut se rassasier, c'est que la chaussette ainsi retournée assume une double fonction de chose enveloppée et de chose enveloppante, qu'elle forme une pochette et son contenu à la fois. Les deux termes de l'opposition, l'extérieur et l'intérieur ne courent aucun danger d'annulation réciproque, aucun d'eux ne se voit supprimé au profit de l'autre. Il ne s'agit pas non plus du renversement radical, irréversible, du système binaire, comme c'était le cas chez Deleuze, mais d'un système à trois éléments où le troisième terme est produit à partir de deux autres. Si les deux éléments se vident, ce n'est que pour former 
un troisième qui, paradoxalement, leur est toujours antérieur. Benjamin, qui parle du culte de la ressemblance chez Proust, écrit :

La ressemblance que nous escomptons entre deux êtres, celle qui nous occupe à l'état de veille, ne touche que superficiellement celle, plus profonde, du monde onirique, dans lequel les événements surgissent, jamais identiques mais semblables : impénétrablement semblables à eux-mêmes. Les enfants connaissent un symbole de ce monde, la chaussette, qui a la structure du monde onirique, lorsque, dans l'armoire à linge, enroulé, il est à la fois "pochette" et "petit cadeau". Et de même qu'ils ne peuvent eux-mêmes se rassasier de changer d'un coup ces deux choses, la pochette et son contenu, en une troisième, la chaussette elle-même, ainsi Proust ne se lassait pas de vider d'un seul coup l'attrape, le moi, pour que toujours à nouveau pût apparaître ce troisième élément, l'image, seule capable de satisfaire sa curiosité, ou, bien plutôt, d'apaiser sa nostalgie ${ }^{24}$.

L'Enfance berlinoise (Armoires) décrit elle aussi la fascination exercée sur l'auteur par les chaussettes retournées. L'admiration pour la forme de la chaussette est ici inséparable de l'admiration pour sa matière, en l'occurrence, la mollesse de sa laine :

Il fallait que je me frayasse un chemin jusqu'au coin le plus reculé, au fond: je tombais alors sur mes chaussettes qui se trouvaient là, empilées et rangées à la manière traditionnelle, c'est-à-dire roulées et enveloppées de telle sorte que chaque paire avait l'aspect d'une petite bourse. Aucun plaisir ne surpassait à mes yeux celui de plonger ma main aussi profondément que possible à l'intérieur... C'était la "chaussette du dedans"... Lorsque je l'avais étreinte avec mon poing et que je m'étais assuré de mon mieux de la possession de la molle masse de laine, commençait la seconde partie du jeu, qui devait se terminer par l'apparition bouleversante de la chaussette... La forme et le contenu, l'enveloppe et l'enveloppé, la "chaussette du dedans" et la bourse sont une seule et même chose, et une troisième aussi, il est vrai : cette chaussette, fruit de leur métamorphose. ${ }^{25}$

31 Il est difficile, on le voit, de surestimer l'importance du textile pour Benjamin, qu'il s'agisse des métamorphoses de l'étoffe (plissement, retournement...) ou de la matière dont elle est fabriquée. L'admiration pour les tissus chez Benjamin est si grande qu'on aurait raison d'affirmer qu'elle est voisine d'un certain fétichisme ou d'une "érotique». Commentant Benjamin, Jean-Louis Déotte parle à cette occasion de l'« importance du gant, du feutre chez ce grand fétichiste : une érotique du gant qui est une érotique du retournement de l'histoire, comme un gant $»^{26}$. Le modèle textile chaussette ou manteau - revient fréquemment sous la plume de Benjamin lorsqu'il parle du rêve. Le rêve est structuré topologiquement: il est une doublure lisse aux couleurs vives qui couvre de l'intérieur un manteau gris et chaud de l'ennui : «L'ennui est une étoffe grise et chaude recouverte, à l'intérieur, d'une doublure de soie aux couleurs vives et chatoyantes. Quand nous rêvons, nous nous roulons dans cette étoffe... Le rêve comme un manteau qu'on ne peut retourner. À l'extérieur, l'ennui gris (du sommeil) $»^{27}$.

Il faut dire que ce jeu du dedans et du dehors est omniprésent chez Benjamin. Tout comme les rêves, les passages urbains "n'ont pas de côté extérieur " ${ }^{28}$. Le passage constitue un pli de l'espace urbain, une poche du dehors, une "rue invaginée ». Pareil à une chaussette qui se retourne sur elle-même, le passage est structuré topologiquement : il est un espace singulier où l'extérieur et l'intérieur, l'individuel et le collectif, le rêve et la veille entrent en rapports de non-opposition. La continuité qui s'établit n'obéit pas aux règles de la géométrie euclidienne. Le passage met en question le modèle bipolaire extérieur/intérieur pour y substituer un rapport topologique, un milieu technique qui se correspond point par point et où il n'y a pas de distances. La 
foule qui traverse le passage se trouve prise dans cette indistinction, en assurant du même coup une "continuité entre englobant et englobé ». Le passage cesse d'être un espace purement extérieur au corps et à la conscience du flâneur qui, en traversant un passage, doit faire siennes la physiologie et la psychologie du lieu. Il doit, tout d'abord, perdre de son intériorité, se plonger dans l'atmosphère ambiante du passage, s'oublier, se laisser hypnotiser par la fantasmagorie: «Tu dois te perdre au sens de t'oublier, faire table rase de ton intériorité, de ton moi psychologique, apprendre à lever les censures, comme le psychanalyste qui doit communiquer d'inconscient à inconscient avec son client, permettre la paradoxale efficacité de la "troisième oreille" : celle de l'écoute flottante $»^{29}$.

En tant qu'il est un "pli du dehors », le passage tel qu'il est décrit par Benjamin n'est pas sans parenté avec une autre notion deleuzienne, celle de pli, qu'on trouve dans le Pli et Foucault, mais aussi dans le Cinéma où il est fréquemment question du " cerveau " ou de la «noosphère », région à structure plissée qui se fait le centre du monde ${ }^{30}$.

Lutte Rouge et Blanche

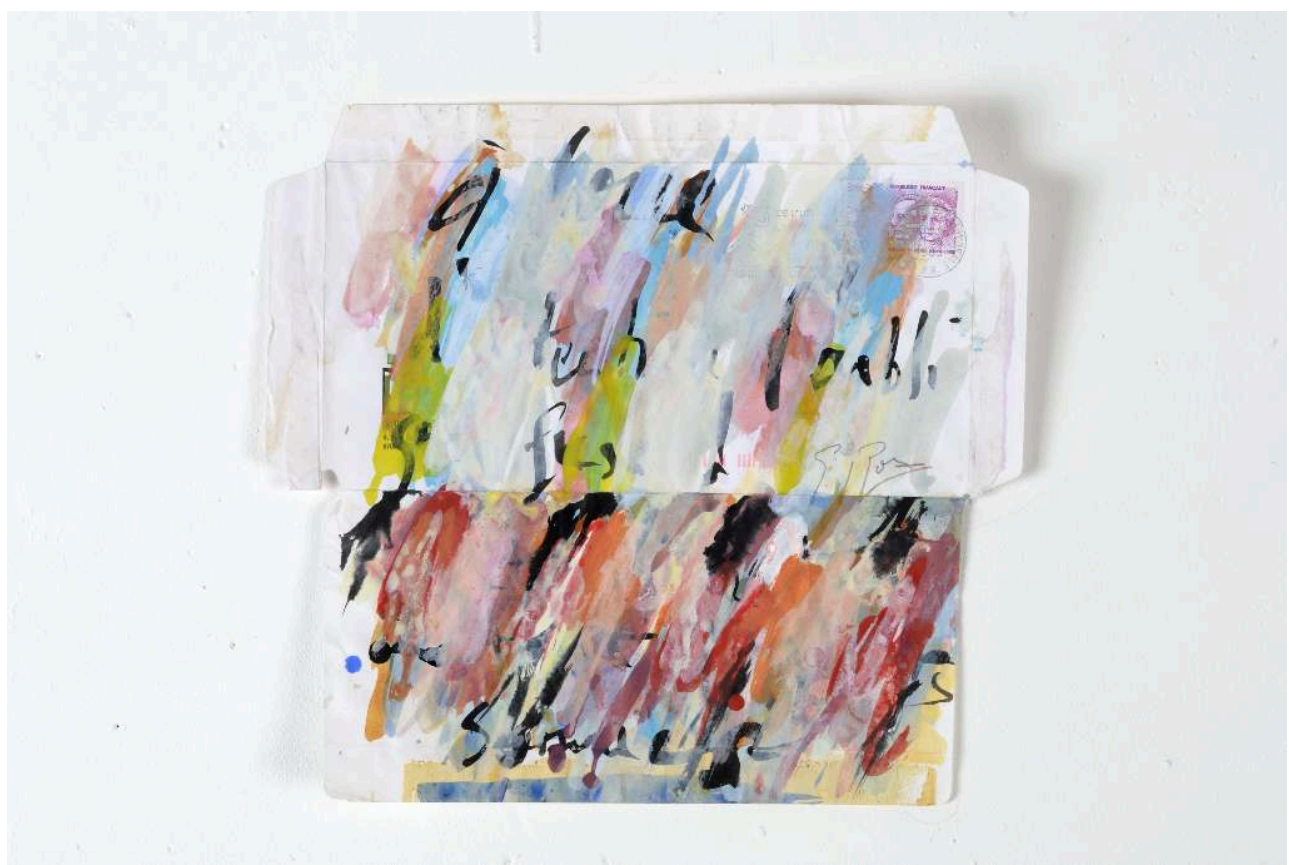

23,3 × 25,3 cm, vinyl encre sur enveloppe dépliée, 1985

Photo Hess

\section{Conclusion}

Comme le montre Déotte, la porosité et le plissage figurent parmi les procédés picturaux préférés de Rœsz. À l'époque où les artistes font des expériences avec le numérique, Rœsz refuse d'être le serf de la communication. Dans une large mesure, son art reste classique, car il n'abandonne pas l'acte de peindre et ses attributs traditionnels : le pinceau, les couleurs, la toile. Avec ces instruments, Rœsz essaie de produire quelque chose de nouveau, en rompant non seulement avec la projection (rupture opérée depuis longtemps par les avant-gardes), mais aussi avec l'inscription : « Rœsz non seulement n'effectue aucun retour à la projection classique inaugurée à la 
Renaissance (stade ultime : Le Grand Verrepar Marcel Duchamp?), mais plus largement ignore ce qui en était encore la condition: l'inscription $»^{31}$. Dans la peinture traditionnelle, un message s'inscrit sur le support, se pose et se fixe là-dessus. Chez Rœsz, le message, la trace colorée vient pénétrer son support, remplir ses pores, l'imprégner. La porosité constitue la condition de ce processus, de l'interpénétration de ces « contraires».

Ce ne sont pas seulement le support et le message qui s'interpénètrent. Une fois posées, les couleurs s'interpénètrent aussi. Rœsz avoue: "je pose souvent une couleur très liquide (cela a son importance) à côté d'une autre et je laisse faire le mélange, la solidarisation hasardeuse $»^{32}$. Au même titre que les couleurs, le corps du peintre participe lui aussi à cette solidarisation. Selon Rœsz, ce qu'il recherche, ce n'est pas l'effet optique, mais « incorporer le corps dans le voir ». Le corps de l'artiste passe sur la toile ou plutôt pénètre la toile comme le font les couleurs.

Cette dimension tactile se fait sentir également dans le choix des surfaces. Le fétichisme des surfaces joue un grand rôle dans le processus de création ainsi organisé. Rœsz semble avoir un goût fort benjaminien pour des matériaux poreux, dont la facture fascine. Rœsz fait son travail « sur plusieurs types de surfaces : du bois, de la toile, du tissu non préparé. Ainsi la couleur peut chercher la couleur contigüe ou imprégner le support, se diffuser, s'étaler, se contracter $"^{33}$.

Le pliage est une autre opération qui permet de dépasser l'opposition des deux surfaces. Il ne s'agit pourtant pas du pliage deleuzien (rien de baroque dans la peinture de Rœsz), mais du pliage benjaminien, qui a quelque chose du jeu d'enfant et qui provoque en nous une joie enfantine : «Arcs-en-ciel, décalcomanies, irisations de bulles de savon, papiers buvard, dilution par quoi le recto et le verso de la toile se confondent, pliages et dépliages aléatoires, peintures blanches les plus translucides possibles. Bref, tout l'univers coloré de la première esthétique de Benjamin $»^{34}$.

\section{BIBLIOGRAPHIE}

Benjamin Walter, Sens unique (précédé de) Enfance berlinoise (et suivi de) Paysages urbains, traduction de l'allemand par Jean Lacoste, $2^{e}$ éd., Paris, Éditions Maurice Nadeau, 1988.

Benjamin Walter, Gesammelte Schriften VII, édition Rolf Tiedemann et Hermann Schweppenhäuser en collaboration avec Christoph Gödde, Henri Lonitz et Gary Smith, Francfort-sur-le-Main, Suhrkamp Verlag, 1989, VII.

Benjamin Walter, Paris, capitale du XIX ${ }^{e}$ siècle : le livre des passages, traduit de l'allemand par Jean Lacoste, Paris, Les Éditions du Cerf, 1997.

Benjamin Walter, Images de pensée, traduction de J.-F. Poirier et J. Lacoste, Paris, Christian Bourgois Éditeur, 1998.

Benjamin Walter, Euvres, tome II, traduction de M. de Gandillac, R. Rochlitz et P. Rusch, Paris, Gallimard, 2000. 
Benjamin Walter, Fragments philosophiques, politiques, critiques, littéraires, traduction de C. Jouanlanne et J.-F. Poirier, Paris, Presses universitaires de France, 2001.

Benjamin Walter, Rêves, édité et postfacé par Burkhardt Lindner, traduit de l'allemand par Christophe David, Paris, Le Promeneur, 2009.

Brüggemann Heinz, Walter Benjamin. Über Spiel, Farbe und Phantasie, Würzburg, Königshausen und Neumann Verlag, 2006.

Deleuze Gilles, Logique du sens, Paris, Éditions de Minuit, 1969.

Deleuze Gilles et Guattari Félix, Mille plateaux : capitalisme et schizophrénie 2, Paris, Éditions de Minuit, 1980.

Déotte Jean-Louis, L'Homme de verre. Esthétiques benjaminiennes, Paris, L'Harmattan, 1998.

Déotte Jean-Louis, L'époque des appareils, Paris, Lignes, 2004.

Déotte Jean-Louis, Walter Benjamin et la forme plastique, Paris, L’Harmattan, 2012.

Déotte Jean-Louis, « Entre trauma et coma », n 13, revue Appareil, 2014.

Derrida Jacques, La vérité en peinture, Paris, Champs-Flammarion, 1978.

Élie Maurice, Lumière, couleurs et nature : l'optique et la physique de Goethe et de la Naturphilosophie, Paris, J. Vrin, 1993.

Gennep Arnold van, Les rites de passage. Étude systématique des rites, Paris, Éditions A. et J. Picard, 1981.

Goethe Johann Wolfgang von, Le traité des couleurs, $3^{e}$ éd. revue, introduction et notes de Rudolf Steiner, textes choisis et présentés par Paul-Henri Bideau, traduction d'Henriette Bideau, Paris, « Triades », 1980.

Kant Immanuel, Critique de la faculté de juger, trad. sous la direction d'Alain Renaut, Paris, Gallimard, coll. « Garnier-Flammarion / Philosophie », 2000.

Lyotard Jean-François, Le Différend, Paris, éditions de Minuit, coll. « Critique », 1983.

Rœsz Germain, « Un coma ça tape où ? incorporer le corps dans le voir », revue Appareil, n 13, 2014.

\section{NOTES}

1. Jean-Louis Déotte, « Entre trauma et coma », n 13, revue Appareil, 2014.

2. Ibid.

3. Walter Benjamin, Gesammelte Schriften VII, édition Rolf Tiedemann et Hermann Schweppenhäuser en collaboration avec Christoph Gödde, Henri Lonitz et Gary Smith, Francfortsur-le-Main, Suhrkamp Verlag, 1989, VII, p. 23.

4. Walter Benjamin, Fragments philosophiques, politiques, critiques, littéraires, traduction de C. Jouanlanne et J.-F. Poirier, Paris, Presses universitaires de France, 2001, p. 130 ; Gesammelte Schriften VII, p. 563.

5. Walter Benjamin, Fragments philosophiques, politiques, critiques, littéraires, op cit., p. 131.

6. Heinz Brüggemann, Walter Benjamin. Über Spiel, Farbe und Phantasie, Würzburg, Königshausen und Neumann Verlag, 2006, p. 169.

7. Immnauel Kant, Critique de la faculté de juger, traduction sous la direction d'Alain Renaut, Paris, Flammarion, coll. « Garnier-Flammarion / Philosophie », 2000, § 14. 
8. Walter Benjamin, Fragments philosophiques, politiques, critiques, littéraires, op. cit., p. 135.

9. Walter Benjamin, Gesammelte Schriften, vol. VII. p. 23.

10. Walter Benjamin, Fragments philosophiques, politiques, critiques, littéraires, op. cit., p. 127.

11. Johann Wolfgang von Goethe, Le traité des couleurs, $3^{\mathrm{e}}$ éd. revue, introduction et notes de Rudolf Steiner, textes choisis et présentés par Paul-Henri Bideau, traduction d'Henriette Bideau, Paris, « Triades ", 1980 et Maurice Élie, Lumière, couleurs et nature : l'optique et la physique de Goethe et de la Naturphilosophie, Paris, J. Vrin, 1993.

12. Jacques Derrida, La vérité en peinture, Paris, Champs-Flammarion, 1978, p. 196.

13. Jean-Louis Déotte, «Entre trauma et coma », op. cit.

14. Germain Rœsz, "Un coma ça tape où? incorporer le corps dans le voir ", revue Appareil, $\mathrm{n}^{\circ} 13,2014$.

15. Voir par exemple Gilles Deleuze et Félix Guattari, Mille plateaux : capitalisme et schizophrénie 2, Paris, Éditions de Minuit, 1980, p. 515, à propos d'Eisenstein.

16. Walter Benjamin, Paris, capitale du XIX $X^{e}$ siècle: le livre des passages, traduit de l'allemand par Jean Lacoste, Paris, Les Éditions du Cerf, 1997, p. 238.

17. Ibid., p. 442. La "compénétration" (die Durchdringung) est une autre notion qui sert à Benjamin pour caractériser le monde de la fantasmagorie. La compénétration dont il s'agit deviendra habituelle dans l'architecture du siècle suivant: selon S. Giedeon, l'architecture de Le Corbusier se caractérise par la même compénétration de l'intérieur et de l'extérieur (ibid., p. 441). 18. Walter Benjamin, Images de pensée, traduction de J.-F. Poirier et J. Lacoste, Paris, Christian Bourgois Éditeur, 1998, p. 11-12.

19. Walter Benjamin, Paris, capitale du XIX ${ }^{e}$ siècle : le livre des passages, op. cit., p. 441.

20. Walter Benjamin, Images de pensée, op. cit., p. 12.

21. Ibid., p. 19. La proximité qui existe entre cette problématique benjaminienne et la fameuse étude d'Arnold van Gennep sur les rites de passage est évidente. [Arnold van Gennep, Les rites de passage. Étude systématique des rites, Paris, Éditions A. et J. Picard, 1981.

22. Gilles Deleuze, Logique du sens, Paris, Éditions de Minuit, 1969, p. 106.

23. Ce motif de la chaussette chez Benjamin est analysé par Jean-Louis Déotte. Voir : Jean-Louis Déotte, Walter Benjamin et la forme plastique, Paris, L'Harmattan, 2012.

24. Walter Benjamin, Euvres, tome II, traduction de M. de Gandillac, R. Rochlitz et P. Rusch, Paris, Gallimard, 2000, p. 140.

25. Walter Benjamin, Sens unique (précédé de) Enfance berlinoise (et suivi de) Paysages urbains,

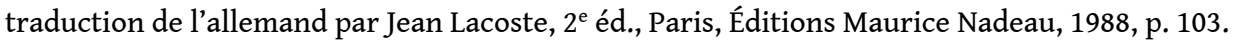

26. Jean-Louis Déotte, L'Homme de verre. Esthétiques benjaminiennes, Paris, L'Harmattan, 1998, p. 25. 27. Walter Benjamin, Rêves, édité et postfacé par Burkhardt Lindner; traduit de l'allemand par Christophe David, Paris, Le Promeneur, 2009, p. 98.

28. Ibid, p. 424.

29. Jean-Louis Déotte, L'époque des appareils, Paris, Lignes, 2004, p. 96.

30. Bien entendu, le contexte esthétique, politique et philosophique du pli chez Deleuze diffère beaucoup des contextes respectifs chez Benjamin. Le pli deleuzien, concept autour duquel s'articule la nouvelle théorie de la subjectivité et de la politique, sert à opérer le dépassement des contraires qui structuraient les théories du sujet dans la métaphysique classique. Deleuze se refuse à penser la subjectivité sous sa forme individuelle et substantielle. Le pli est un espace sans extériorité, une poche, une zone d'intériorité formée à partir de l'extérieur au moyen d'une inflexion. Résultats d'une inflexion, les deux régions - le dedans et le dehors - ne s'opposent plus, elles font un et sont inséparables. Deleuze fait valoir la richesse du paradigme du plissage. Selon lui, "plier-déplier ne signifie plus simplement tendre-détendre, contracter-dilater, mais envelopper-développer, involuer-évoluer ", d'où la diversité et le grand nombre de plis qui se rencontrent. Cela signifie, dans le contexte politique qui est celui de Deleuze, que le nombre de plis - chaque pli constituant l'envers des rapports de pouvoir et devant sa formation à leur 
inflexion - correspond au nombre des sociétés hiérarchisées. Finalement, la formation d'une zone d'intériorité se fait dans le champ politique, et la subjectivité, c'est-à-dire le pli, est un sousproduit des rapports de pouvoir.

31. Jean-Louis Déotte, « Entre trauma et coma », op. cit.

32. Germain Rœsz, « Un coma ça tape où ? Incorporer le corps dans le voir », op. cit.

33. Ibid.

34. Jean-Louis Déotte, «Entre trauma et coma », op. cit.

\section{RÉSUMÉS}

Ce texte s'interroge sur la possibilité de l'esthétique benjaminienne en peinture. À la suite des travaux de J.-L. Déotte, il essaie de dégager quelques concepts importants pour l'esthétique du jeune Benjamin, tels que la couleur, le pore, le pli, le tissu, et de montrer comment ces concepts font leur apparition dans la peinture de G. Rœsz.

\section{INDEX}

Mots-clés : appareils, peinture, couleur, interpénétration, pore, pli, fétichisme, tissu

\section{AUTEUR}

\section{DENIS SKOPIN}

Maître de conférences, Université de Saint-Pétersbourg 